Research paper

\title{
The effectiveness of modified, group-based CBT for dementia worry among Chinese elders
}

\author{
Qiuling $\mathrm{An}^{\mathrm{a}}$, Kaipeng Wang ${ }^{\mathrm{b}, *}$, Fei Sun ${ }^{\mathrm{c}}$, Anao Zhang ${ }^{\mathrm{d}}$

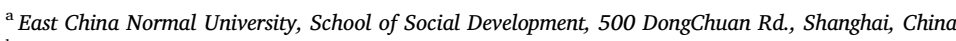 \\ ${ }^{\mathrm{b}}$ University of Denver, Graduate School of Social Work, Denver, CO, USA \\ ${ }^{\mathrm{c}}$ Michigan State University, School of Social Work, East Lansing, MI, USA \\ ${ }^{\mathrm{d}}$ University of Michigan, School of Social Work, Ann Arbor, MI, USA
}

A R T I C L E IN F O

\section{Keywords:}

Anxiety

Cognitive-behavioral therapy

Cultural modification

Experimental design

\begin{abstract}
A B S T R A C T
Objectives: Dementias are highly prevalent among Chinese elders. This study examined the effectiveness of a modified group cognitive behavioral therapy (CBT) on dementia worry among Chinese older adults. Methods: Eighty-two older adults recruited from four elder group homes were randomly assigned to either intervention or control group. The intervention group $(n=44)$ received eight weekly 60 -minute face-to-face CBT, while the control group $(\mathrm{n}=38)$ received treatment-as-usual.

Results: Outcomes indicated that the modified group CBT has significantly reduced dementia worry and culturally biased beliefs about dementia $(p<.001)$. Study findings supported both statistically and clinically significant effect of modified group CBT on dementia worry $[g=-1.52,95 \%$ CI $(-2.01,-1.03)]$ and biased beliefs about dementia $[g=-.95,95 \%$ CI $(-1.40,-.49)]$.

Discussion: The culturally adapted CBT is promising in alleviating worries and anxiety over dementia among Chinese older adults. Future research needs to include larger samples and participants from different regions to replicate findings.
\end{abstract}

\section{Introduction}

Population aging has contributed to increased prevalence of dementia globally. Worldwide, about 50 million people are living with dementia in 2019, with the expected growth to 65.7 million by 2030 and 115.4 million by 2050 (Prince et al., 2013). In China, the number of people with dementia in 2010 (9.19 million) almost tripled since 1990 (3.68 million) (Chan et al., 2013). As Chinese older adults ( $\geq 65$ years old) become increasingly aware of the prevalence and consequences of dementia, dementia worry has become a salient psychosocial concern among them (Lam et al., 2019; Zhang, 2018).

Dementia worry, also termed as anxiety or fear related to dementia, is an anxiety-related response to the possibility of developing dementia (Molden and Maxfield, 2017). Dementia worry represents an important yet underexplored health concern for geriatric populations, including Chinese older adults (Kessler, Bowen, Baer, Froelich, and Wahl, 2012; Sun, Gao and Coon, 2013). Previous research has indicated dementia as the leading cause of fear second to cancer in the adult population (Blendon et al., 2011). Studies focusing on Chinese populations found that more than half of older adults are "very concerned" about their declined memory as an early sign of dementia (Laditka et al., 2011; Liang and An, 2018). While dementia worry may permeate across older populations regardless of their racial/ethnic background, Chinese culture related beliefs of dementia make Chinese older adults more susceptible to dementia worry (Zeng et al., 2015). Specifically, studies have found that older Chinese adults equate getting dementia as becoming a burden to their family members (e.g., Guo, 2003; Lam et al., 2019). This is in large part due to the filial piety culture in China which anticipates younger family members to take care of older adults when they are ill, including having dementia (Chang, Schneider, and Sessana, 2011; Yu et al., 2016). Therefore, dementia worry has become a salient concern among Chinese older adults.

Although vigilance to one's symptoms could lead to early detection of dementia, hypervigilance to and excessive anxiety about normal agerelated cognitive changes are likely to contribute to misdiagnosis of dementia, trigger inappropriate treatment, and negatively affect an individual's psychosocial and relational support system (Cutler and Hodgson, 1996; Kinzer and Suhr, 2015). Furthermore, in addition to \footnotetext{
7100 .

E-mail address: Kaipeng.Wang@du.edu (K. Wang).
} 
poor psychosocial outcomes, excessive anxiety has also been associated with higher risk of developing dementia. One study reported a higher level of anxiety was associated with increased risk for overall cognition decline and dementia (Wilson, Begeny, Boyle, Schneider, and Bennett, 2011). In addition, a twin study revealed that those with high anxiety were $48 \%$ more likely to develop dementia than their counterparts with low anxiety (Petkus et al., 2016). Results of this study not only implied a possible genetic linkage between anxiety and dementia but also attested to an empirical association between anxiety and the development of dementia while accounting for other genetic factors. A recent systematic review and meta-analysis further affirmed that anxiety is associated with greater risk for cognitive impairment and dementia (Gulpers et al., 2016). Despite an accumulating body of literature, empirical evidence supporting the direct connection between dementia worry and dementia risk remains preliminary. Though there lacks a definitive conclusion between anxiety and the risk of developing dementia, ample studies have supported the impact of dementia worry on older adults' cognitive health and quality of life (e.g., Kessler et al., 2012; Kinzer and Suhr, 2016; Seignourel et al., 2008). Therefore, it is essential to explore ways to reduce dementia worry - a unique form of health anxiety associated with dementia.

\subsection{Theoretical framework}

To date, cognitive-behavioral therapy (CBT) is one of the best evidence-based psychosocial treatments for anxiety disorders (Cuijpers et al., 2014). Building on Beck's (1985) cognitive model of anxiety, Warwick and Salkovskis (1990) developed a cognitive-behavioral theory specific for health anxiety. Specifically, anxiety is proportional to $\frac{\text { severity of threat } x \text { severity of consequences }}{\text { likelihood of rescue }+ \text { perceived coping factors }}$ (Warwick, 2004, p. 80). If an adult believes his/her occasional memory lapses are early signs of dementia (i.e., severity of threat: I will have dementia) and wrongfully perceives dementia as an acute condition that leads to amnesia (i.e., severity of consequences: I am not going to remember anyone soon), this individual is expected to report a very high level of anxiety. Similarly, one's dementia worry is likely to decrease if the individual understands ways to slow the progression of dementia (i.e., likelihood of rescue: even if I have it, I still have good chances of many lucid years and good quality of life) and to reduce the risk for dementia (i.e., perceived coping factors: I can do more exercises and socialize more frequently). Therefore, interventions should also improve knowledge and belief about dementia to address dementia worry.

Additionally, a core ingredient of CBT - behavioral activation - is also critical in reducing individuals' health anxiety, including dementia worry. Strategies of behavioral activation treating anxiety will help clients rationalize the consequences of anxiety, provoking thoughts/ incidents that are positive and rewarding to clients coping. This would allow clients to have more opportunities to engage with situations and thoughts that are reinforcing and meaningful (Boswell, 2013; Boswell, Iles, and Gallagher, 2017). For example, if an older adult thinks jogging is a protective factor for dementia, encouraging that client to jog and reflect on a regular basis is likely to reduce his dementia through behavioral activation.

\subsection{Existing empirical evidence}

Guided by the above-mentioned cognitive behavioral model for health anxiety, CBT interventions specifically target those core cognitive and behavioral components to reduce older adults' health anxiety. Existing studies have reported that various forms of CBT interventions can effectively reduce worries and anxiety due to different health conditions. For example, Clark and colleagues (1998) found cognitive therapy has a lasting effect for hypochondriasis (i.e., unwarranted fear about a disease) treatment. More recently, a few randomized controlled trials reported both individual-based (Barsky and Ahem, 2004) and group-based (Hedman et al., 2010) CBT interventions are effective for hypochondriasis in a variety of settings. Finally, a meta-analysis of CBT for hypochondriasis/health anxiety (Olatunji et al., 2014) revealed that CBT significantly improves participants' health anxiety in comparison to no-treatment control, with a large treatment effect $d=.95$, which indicated meaningful clinical improvements in participants' health anxiety.

While numerous trials of CBT have been tested for anxiety and worry among people with dementia or their caregivers (Cooper, Balamurali, Selwood, and Livingston, 2007; Spector et al., 2015), to our knowledge, few studies have specifically focused on older adults with dementia worry - a unique yet highly relevant form of anxiety for older adults. Additionally, our review of literature has identified limited study has applied and tested CBT interventions for dementia worry among Chinese older adults. The lack of such investigation is partly due to challenges associated with modifying CBT for Chinese populations (e.g., Guo and Hanley, 2015). While it is reasonable to expect the cognitive-behavioral theory for health anxiety to apply to Chinese populations, adaptations must be made for Chinese older adults to ensure CBT's fidelity and optimal effect (Hui and Zhihui, 2017).

\subsection{Modifying group CBT interventions for Chinese older adults}

Although the conceptual literature on CBT adapted for Chinese populations is still accumulating, some consensuses on best practices are available across various models of modification (Chen, and Davenport, 2005; Hwang, 2006). First, CBT's change theory and session structure should remain unchanged (Rathod, Phiri, and Naeem, 2019), as an intact session structure and close adherence to CBT's change theory sustains CBT's fidelity (Hwang, 2006; Hwang et al., 2015). The five core CBT sessions should include (1) introduction to CBT and building therapeutic relationships, (2) cognitive restructuring, (3) relaxation training, (4) behavioral activations, and (5) relapse prevention. The order of these sessions may vary depending on specific mental disorder and treatment setting, though.

Second, therapeutic relationships and group dynamics in group CBT should be formed via culturally competent practice with Chinese populations (Chen and Davenport, 2005). As a group CBT therapist primarily serves as a facilitator and educator, this "teacher" role entails a sense of authority that may inhibit disagreement from group participants from being openly expressed (Tung, 1991). Therefore, it is important to clarify to group participants of the therapeutic process and the role of the therapist prior to intervention. Besides, the CBT therapist needs to provide higher-than-usual dosage of psychosocial education without making group members feel compelled to participate using a more directive approach. Asians, including Chinese, tend to have lower tolerance for ambiguity and prefer practical and immediate problem solving (Leong, 1986). Therefore, instead of predominantly using Socratic questioning techniques (open-ended "why" and "what" type of questions), more directive questions and communications with Chinese older adults should be encouraged to solicit ideas from group members (Chen, 1995).

Finally, the cognitive restructuring, skill-training, and behavioral activation components of CBT need to be modified to be culturally relevant to Chinese older adults (Hwang, 2006). Rather than directly disclosing an older individual's irrational thinking patterns, combining psychoeducation and cognitive restructuring can be more effective to achieve cognitive restructuring (Hays, 2009). Additionally, TaiChi, as a behavioral activation component that has shown benefits to cognitive health and popularity among Chinese older adults, is recommended exercise to motivate and engage Chinese older adults (Wayne et al., 2014). Additionally, TaiChi is a well-validated approach to alleviate older adults' psychological distress (Wang et al., 2010), with studies supporting TaiChi in reducing anxiety among individuals with dementia (Tadros et al., 2013; Wang, Collet, and Lau, 2004). 
Consequently, we expect TaiChi would be a beneficial exercise for individuals with dementia worry.

\subsection{The present study}

Grounded in the cognitive behavioral theory for health anxiety and the literature on modified CBT for Chinese older adults, this present study evaluates the effectiveness of a modified group CBT intervention on Chinese older adults' dementia worry. We hypothesize that modified group CBT intervention will reduce dementia worry, improve knowledge of dementia, and reduce false belief about dementia among participants.

\section{Methods}

This study used a pre-test-post-test randomized controlled trial (RCT) design. Participants received baseline evaluation and post-intervention assessment immediately after treatment completion. The study was reviewed and approved by the Institutional Review Board at "blinded for review". The study was not preregistered in an independent institutional registry due to privacy protection and local legal restrictions.

\subsection{Setting, eligibility criteria, and recruitment procedures}

This RCT recruited participants from four elder group home facilities, one private facility (Site C) and three public facilities (Sites A, B, and D) in Shanghai, China during a 10-month period (August 2017 May 2018). A total of 700 older adults were residing in the four facilities. Eligibility criteria included: 65 years or older, normal cognitive function evidenced by a score of 22 or higher using the Beijing version of the Montreal cognitive assessment (Li et al., 2016; Yu, Li, and Huang, 2012). While the recommended cut-off score for the cognitive assessment is 26 or higher, we adopted Yu and colleagues' (2012) suggestion to obtain cultural sensitivity and specificity for Chinese older adults using the 22 cut-off score. Participants also needed to be physically healthy and feel anxious about dementia indicated by a score of 22 or higher using the Dementia Worry Scale (Sun, et al., 2013). Participants were recruited by the Social Service Department of each facility or through self-referrals.

\subsection{Randomization and blinding}

Consented eligible participants were randomized into either group CBT intervention or no-treatment control. A computerized random number generator created a list of random non-repetitive integer numbers ranging from 1 to 100, which then were assigned to participants as they entered the trial. Participants with an odd number were assigned to the group CBT. Group facilitators could not be blinded given the nature of the intervention. Participants and outcome assessors were blinded to participants' group assignment.

\subsection{Modified group CBT intervention}

This modified group CBT treatment modules were developed in line with the cognitive behavioral theory of anxiety, with key intervention components mapping onto different components of the theory (See Fig. 1). In general, participants received eight sessions of group CBT sessions, 60 minutes per session, across a two-month period. The development of the modified group CBT intervention was based on Norton's (2012) transdiagnostic group CBT for anxiety, and included core CBT components: (1) psychoeducation about CBT, causes of dementia worry, and knowledge about dementia, (2) physical activity and behavioral activation through TaiChi training, (3) cognitive restructuring in conjunction with psychoeducation (e.g., given the updated knowledge about brain health, how to think about dementia differently?), and (4) summary and relapse prevention (See Fig. 1). To develop group CBT content that is culturally appropriate for Chinese older adults, the research team conducted focus groups to help inform the development of treatment content (Liang and An, 2018). For example, older adults suggested TaiChi as the preferred group activity and homework assignment. They also indicated strong preference of indoor exercises to improve their brain function. The treatment manual of this modified CBT group intervention was available upon reasonable request to the first author.

\subsection{No-treatment control group}

To evaluate whether participant changes resulted from modified group CBT intervention or from the amount of time spent with service providers, the protocol for no-treatment control matched that of the modified group CBT. Like modified group CBT, participants in the notreatment control group met eight times over the course of 10 weeks. These group sessions primarily included opportunities for socialization and group activities. Content relevant to dementia was included but not as the focus of any group session. Facilitators were instructed to refrain from providing any therapeutic support to participants in the notreatment control group.

\subsection{Training group facilitators and assessing fidelity}

Four graduate-level social work interns were selected and trained to become group facilitators for this study (two for group CBT and two for no-treatment control). All four facilitators received one semester training in group facilitation and CBT. Two facilitators for modified group CBT were further trained by an experienced clinician (over eight years of experience) in delivering group CBT with older adults for health anxiety as well as by a certified Taichi trainer. The two CBT facilitators were evaluated in group facilitation role play activities and received satisfactory scores before starting clinical contact. All four facilitators received on-going weekly supervision by the study's PI who has training and clinical experience in psychology. Specific attentions during the training and on-going supervision were given to the facilitators' use of more directive communication language, facilitating productive group interactions, and working with resistant or disruptive group participants.

\subsection{Data collection and outcome measures}

Participants completed assessments at baseline and immediately upon treatment completion. Background variables included basic health information and socio-demographics such as age, gender, marital status, and education.

\subsubsection{Primary outcome}

Dementia worry was measured by a nine-item scale adapted from the Perceived Threat of Alzheimer's scale (Robert and Connell, 2000). This scale has achieved satisfactory reliability and validity among older Chinese Americans (Sun et al., 2013). Example of questions included "I could not sleep because of worry about dementia", and "I often think about developing dementia." Participants rated from 1 (completely disagree) to 5 (completely agree). Higher scores indicate more worries about dementia. The Cronbach's alpha of this scale was 0.69 for pretest and 0.80 for posttest.

\subsubsection{Secondary outcomes}

Knowledge of dementia contained 24 "true" or "false" questions adapted from the Alzheimer's Disease Knowledge Scale (Carpenter et al., 2009) in line with the updated evidence supported facts about Alzheimer's disease and related dementia (Sun et al., 2013). Higher scores indicated higher levels of knowledge of Alzheimer's disease and related dementias. In this study, the KR20 coefficient of this 
Sessions

\begin{tabular}{|l|}
\hline Session 1: Introducing CBT \\
\hline
\end{tabular}

Session 2: Psychoeducation and Cognitive Restructuring 1

Session 3: Psychoeducation and Cognitive Restructuring 2

Session 4: Physical Exercise and Behavioral Activation 1

Session 5: Physical Exercise and Behavioral Activation 2

Session 6: Integrative Session 1

Session 7: Integrative Session 2

Session 7: Integrative Session 2

Session 8: Summary and Relapse Prevention
Specific Purposes and Content

1. Ice breaking, setting group rules

2. Brief introduction to group CBT

3. Expectations and review of future sessions

1. Psychoeducation about brain health

2. Psychoeducation about dementia

3. Psychoeducation about dementia worry

1. Think differently with knowledge about brain health

2. Coping with anxiety associated with dementia worry

1. Taichi training

1. Taichi training

2. Discussing how practicing Taichi may help prevent dementia

3. Discussing how practicing Taichi may reduce dementia worry

1. Brief review of psychoeducational content of brain health and dementia worry

2. Brief review of Taichi

3. Connecting psychoeducation and Taichi exercise

1. Linking psychoeducation, Taichi exercise with dementia worry and reducing anxiety

2. Discussing challenges and troubleshooting for setbacks

1. Comprehensive review of content and participants' progress

2. Discussing relapse prevention about dementia worry

3. Discussing sustainability about continuing Taichi exercise

Fig. 1. Brief outline of the modified group CBT sessions.

scale was 0.74 for pretest and 0.61 for posttest.

Cultural beliefs of dementia were assessed by an 11-item dementia belief scale that has been validated on older Chinese Americans (Sun et al., 2013; Shi, Sun, Liu, and Marsiglia, 2018). Participants rated the degree on five-point Likert scale to which they agreed with a series of statements constructed around two major beliefs about dementia: "dementia as a normal part of aging" and "dementia as a stigmatized mental illness". Higher scores indicated more culturally biased beliefs toward dementia. In this study, the Cronbach's $\alpha$ is 0.85 for pretest and 0.82 for posttest.

\subsection{Statistical methods and power analysis}

A minimal total sample size of 45 participants per group $(\mathrm{N}=90)$ is required to achieve an $80 \%$ power to detect a moderate treatment effect size $(d=0.6)$ with independent t-tests for two groups at a two-tailed $\alpha$ level of 0.05. We used an estimated effect size of $d=0.6$ to power our study based on effect sizes reported from existing meta-analysis of CBT for anxiety disorders (Carpenter et al., 2018; Hofmann and Smits, 2008; Watts et al., 2015). This study reached out to 131 participants and resulted in a final analytical sample of 82 participants. First, we compared descriptive statistics of demographic and baseline characteristics between- the modified CBT group and the no-treatment control group. We further compared those variables between participants who completed the study and those who dropped out within each treatment group. Second, we used multiple imputation with chained equation (MICE) approach as intent-to-treat analysis to address missing values. We generated 20 multiple imputed datasets separately for the treatment group and no treatment group, and obtained the pooled results from all imputed datasets (Puma, Olsen, Bell, and Price, 2009; Royston and White, 2011). Based on the imputed datasets, we conducted regression by regressing changes in outcomes on treatment status to examine whether changes in outcomes (the difference between the post-test and pre-test) were statistically different between the two groups. We calculated Hedges' $g$ to assess the practical significance, i.e. effect size, of the modified CBT treatment. Third, for sensitivity analysis, we conducted ordinary least squares (OLS) regression to examine the treatment effects after adjusting for sociodemographic and baseline characteristics following MICE. We also used sites as dummy variables to account for clustering effect. Given the methodological debates on whether change score or posttest score should be used as to evaluate the treatment effect (e.g., Fu and Holmer, 2015), we also conducted the above sensitivity analysis using post-test scores as the outcome after multiple imputation adjusting for the pre-test scores and covariates to confirm the robustness of our findings. Finally, we conducted heterogeneity test to examine whether the treatment effects differed by sociodemographic or baseline characteristics following MICE, through 


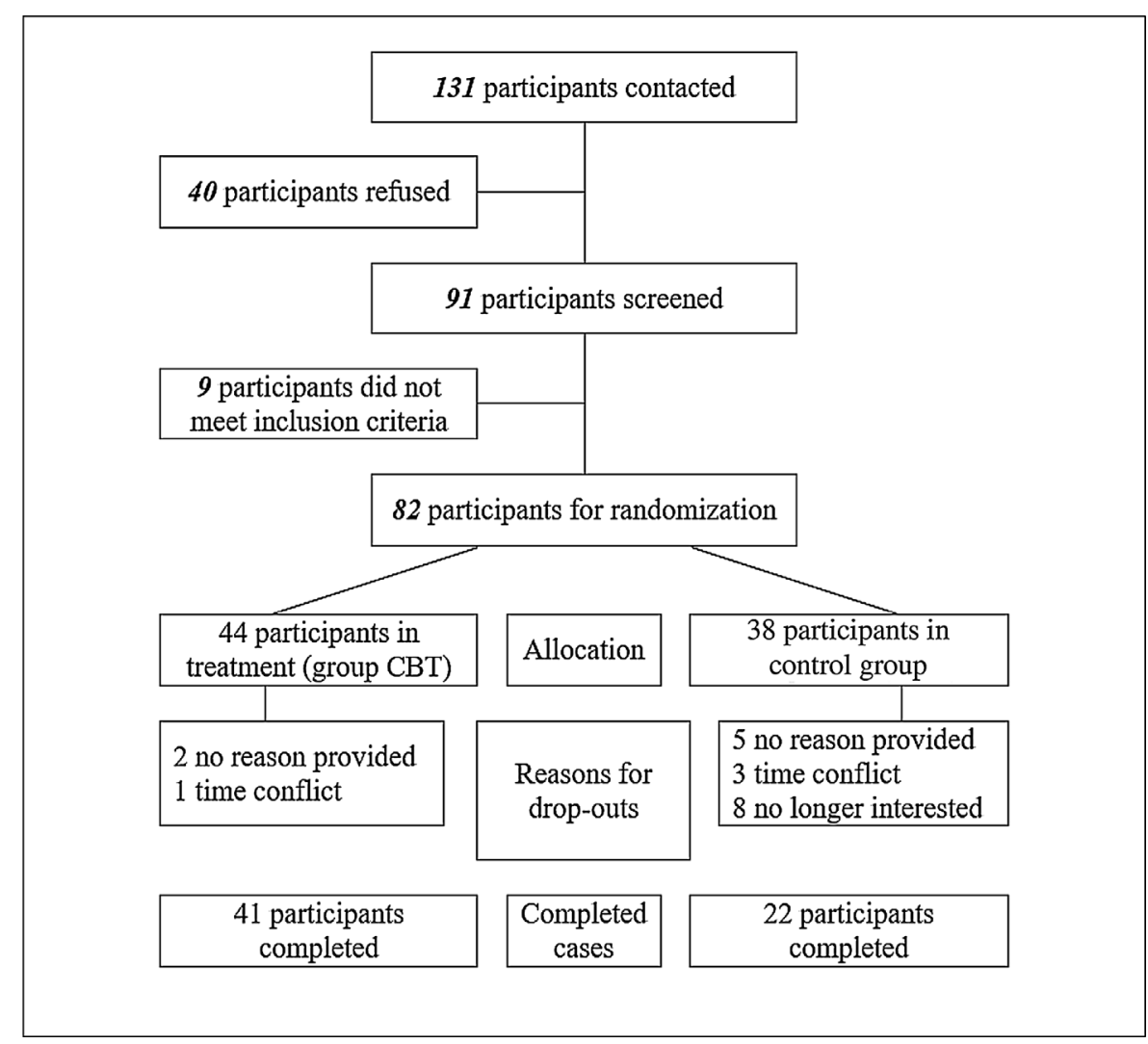

Fig. 2. CONSORT flowchart of participant recruitment, randomization and retention.

testing the interaction terms between treatment assignment and each covariate after adjusting for all sociodemographic and baseline characteristics.

\section{Results}

\subsection{Recruitment, enrollment, and retention}

Over the course of 10 months, the research team contacted 131 potentially eligible participants, among whom, 9 were excluded because of non-eligibility and 40 refused to participate. Reasons for decline to participate mainly included "not interested in this topic" $(n=27)$ and "time conflict" $(n=10)$. A final number of 82 participants entered the trial for randomization. During the trial, three participants dropped out of the modified group CBT whereas 16 participants dropped out of the no-treatment control group. Half of the participants ( $\mathrm{n}=8$ ) who dropped out of the no-treatment control reported "no longer interested" as their reasons for drop out. Fig. 2 presents the CONSORT flowchart of recruitment, treatment assignment, and retention. Participants all gave informed consent to participate in this study.

\subsection{Baseline characteristics}

Table 1 presents descriptive statistics of participant demographic and baseline information. Participants' $(\mathrm{N}=82)$ age averaged at 85.11 years old $(S D=4.59)$ and over half were female $(\mathrm{n}=56,68.29 \%)$. Over half of the participants were born in Shanghai $(n=52,63.41 \%)$. Most of the participants were not married ( $n=71,86.59 \%)$, including 70 widowed and one never married. Over $20 \%(n=19,23.17 \%)$ of the participants had a high school diploma or higher, leaving the majority of participants with middle school education $(n=27,32.95 \%)$ or with elementary school education or below ( $\mathrm{n}=36,43.9 \%)$. Most participants reported no religious beliefs ( $n=74,90.24 \%$ ). Close to half
Table 1

Participants' demographic and baseline characteristics $(\mathrm{N}=82)$

\begin{tabular}{lll}
\hline & $\mathrm{M} / \mathrm{SD}$ & $\mathrm{n}(\%)$ \\
\hline Participants' age & $85.11 / 4.59$ & \\
Participants' gender (female) & & $56(68.29)$ \\
Marital status & & \\
$\quad$ Currently married & & $11(13.41)$ \\
$\quad$ Currently not married & & \\
Education & & $36(46.59)$ \\
$\quad$ Elementary school or lower & & $27(32.90)$ \\
Middle school & $19(23.17)$ \\
High school or higher & & $8(9.76)$ \\
Having religious belief (yes) & & $52(63.41)$ \\
Born in Shanghai (yes) & $3(3.80)$ \\
Self-rated health & $22(27.85)$ \\
$\quad$ Very poor & & $34(43.04)$ \\
Poor & & $18(22.78)$ \\
Fair & & $2(2.53)$ \\
Good & & \\
Excellent & & $42(51.22)$ \\
Facilities & & $10(12.20)$ \\
Site A & & $16(19.51)$ \\
Site B & & $14(17.07)$ \\
Site C & & \\
Site D & & \\
Dementia worry & & \\
Knowledge about dementia & & \\
Cultural beliefs about dementia & 27.49/5.12 \\
\hline
\end{tabular}

indicated fair self-rated health $(\mathrm{n}=34,43.04 \%)$. For baseline clinical evaluation, participants reported an average of $27.49(S D=5.12)$ for dementia worry, 11.66 (SD $=4.12$ ) for knowledge about dementia, and 33.22 (SD $=9.08$ ) for cultural beliefs about dementia.

Between-group analyses were conducted to determine any difference in demographic and baseline characteristics in participants between two groups (Table 2). Participants were similar in all 
Table 2

Comparison of demographic and baseline evaluation between intervention and control groups, and between completers and dropouts within each groups ${ }^{\text {a }}$

\begin{tabular}{|c|c|c|c|c|c|}
\hline & \multicolumn{2}{|l|}{ Modified group CBT } & \multicolumn{2}{|l|}{ No-treatment control } & \multirow[b]{2}{*}{ Between-group difference ( $\mathrm{t}$ test or $\chi^{2}$ ) } \\
\hline & Completers $(n=41)$ & Dropouts $(n=3)$ & Completers $(\mathrm{n}=22)$ & Dropouts $(\mathrm{n}=16)$ & \\
\hline Participants' age & $84.51 / 5.19$ & $89.67 / 4.93$ & $84.73 / 3.87$ & $86.58 / 2.81$ & $\mathrm{t}(74)=-.77$ \\
\hline Participants' gender (female) & $58.54 \%$ & $66.67 \%$ & $72.73 \%$ & $87.50 \%$ & $\chi^{2}(1)=3.71$ \\
\hline Marital status (currently married) & $19.51 \%$ & $33.33 \%$ & $9.09 \%$ & $0 \%$ & $\chi^{2}(2)=5.04$ \\
\hline Education & & & & & $\chi^{2}(3)=1.61$ \\
\hline Elementary school or lower & $46.34 \%$ & $0 \%$ & $63.64 \%^{\mathrm{b}}$ & $18.75 \%^{\mathrm{b}}$ & \\
\hline Middle school & $34.15 \%$ & $66.67 \%$ & $22.73 \%^{\mathrm{b}}$ & $37.50 \%{ }^{\mathrm{b}}$ & \\
\hline High school or higher & $19.51 \%$ & $33.33 \%$ & $13.64 \%^{\mathrm{b}}$ & $43.75 \%^{\mathrm{b}}$ & \\
\hline Having religious belief (yes) & $12.20 \%$ & $33.33 \%$ & $4.55 \%$ & $6.25 \%$ & $\chi^{2}(1)=1.62$ \\
\hline Born in Shanghai (yes) & $60.98 \%$ & $66.67 \%$ & $77.27 \%$ & $50.00 \%$ & $\chi^{2}(1)=.17$ \\
\hline Self-rated health & & & & & $\chi^{2}(4)=8.48$ \\
\hline Very poor & $0 \%$ & $0 \%$ & $4.55 \%$ & $12.50 \%$ & \\
\hline Poor & $18.42 \%$ & $33.33 \%$ & $40.91 \%$ & $31.25 \%$ & \\
\hline Fair & $50.00 \%$ & $33.33 \%$ & $36.36 \%$ & $37.50 \%$ & \\
\hline Good & $26.32 \%$ & $33.33 \%$ & $18.18 \%$ & $18.75 \%$ & \\
\hline Excellent & $5.26 \%$ & $0 \%$ & $0 \%$ & $0 \%$ & \\
\hline \multicolumn{6}{|l|}{ Facilities } \\
\hline Site A & $41.47 \%$ & $100 \%$ & $36.36 \%^{\mathrm{b}}$ & $87.50 \%{ }^{\mathrm{b}}$ & \\
\hline Site B & $19.51 \%$ & $0 \%$ & $0 \%{ }^{\mathrm{b}}$ & $12.50 \%^{\mathrm{b}}$ & \\
\hline Site C & $19.51 \%$ & $0 \%$ & $36.36 \%^{\mathrm{b}}$ & $0 \%^{\mathrm{b}}$ & \\
\hline Site D & $19.51 \%$ & $0 \%$ & $27.27 \%^{\mathrm{b}}$ & $0 \%^{\mathrm{b}}$ & $\chi^{2}(3)=3.56$ \\
\hline Dementia worry & $28.29 / 4.71$ & $29.67 / 5.13$ & $27.73 / 4.64^{\mathrm{b}}$ & $24.69 / 6.18^{\mathrm{b}}$ & $\mathrm{t}(80)=1.73^{*}$ \\
\hline Knowledge about dementia & $12.29 / 3.32^{\mathrm{b}}$ & $6.67 / 2.08^{\mathrm{b}}$ & $14.32 / 3.96^{\mathrm{b}}$ & $7.31 / 1.74^{\mathrm{b}}$ & $\mathrm{t}(80)=.59$ \\
\hline Cultural beliefs about dementia & $34.27 / 9.46^{\mathrm{b}}$ & $20.00 / 4.58^{b}$ & $36.32 / 7.77^{b}$ & $28.75 / 6.77^{b}$ & $\mathrm{t}(80)=.08$ \\
\hline
\end{tabular}

${ }^{\text {a }}$ For descriptive statistics, we present Mean/Standard Deviation for continuous outcomes and \% for categorical outcomes.

b Indicates the difference between completers and non-completers (drop-out) was statistically significant at .05.

$* p<.05 a$.

demographic and baseline characteristics except for dementia worry. Specifically, participants in the no-treatment control group reported significantly lower dementia worry than their counterparts in the intervention group $[t(80)=1.73, p<.05]$ at baseline. In the intervention group, those who completed the intervention reported significantly higher scores in knowledge about dementia $[t(43)=-2.88, p<.01]$ and higher cultural beliefs about dementia $[t(43)=-2.57, p<.01]$ at baseline than those who dropped out of the study. In the no-treatment control group, those who completed no-treatment control had higher scores in dementia worry $[t(36)=-1.74, p<.05]$, more knowledge about dementia $[t(36)=-6.61, p<.001]$, and higher cultural beliefs about dementia $[t(36)=-3.12, p<.01)]$ than those who dropped out.

\subsection{Treatment effect and effect sizes}

Table 3 presents results of between-group comparison using unadjusted treatment effect, treatment effect after adjusting for demographic and baseline characteristics, and Hedges' $g$ effect size. The unadjusted treatment effect revealed participants in the intervention group had a significantly greater reduction in their dementia worry $(b=-10.02, p<.001)$ and biased cultural beliefs about dementia $(b=-8.75, p<.001)$ than their counterparts in the no-treatment control group.

Between-group treatment effect sizes indicated large treatment effect of modified group CBT as compared to no-treatment control. Specifically, participants in the intervention group on average reported
1.49 standard deviation $(g=-1.49, p<.001)$ greater reduction in dementia worry and .95 standard deviation $(g=-.87, \mathrm{p}<.001)$ greater reduction in culturally biased beliefs about dementia than their counterparts in no-treatment control. The treatment effect of modified group CBT for participants' knowledge about dementia was not statistically significant in comparison to no-treatment control $(g=-0.30, p=0.77)$.

\subsection{Sensitivity analysis}

The treatment effects after adjusting for baseline and demographic characteristics were $b=-9.47, p<.001$ for dementia worry, $b=-0.30$, $p=0.76$ for knowledge about dementia, and $b=-8.65, p<.001$ for false beliefs about dementia, respectively.

\subsection{Heterogeneity test}

The treatment effect of modified group CBT on dementia worry differed significantly depending on education background. The treatment effect on dementia worry was strongest for participants with elementary school education or below $(b=-12.38, p<.001)$, followed by participants with middle school education $(b=-7.09, \mathrm{p}<.001)$. The treatment effect on dementia worry was not statistically significant for those with high school education or above, $b=-2.58, p=.32$.

We found that the treatment effect of modified group CBT on biased belief about dementia differs significantly by age and baseline biased belief. The treatment effect significantly became less pronounced with

Table 3

Results of treatment effect size and between-group comparison

\begin{tabular}{|c|c|c|c|c|c|c|c|c|c|}
\hline & \multicolumn{3}{|c|}{ Modified group CBT } & \multicolumn{3}{|c|}{ No-treatment control } & & & \\
\hline & Pre-M/SE ${ }^{a}$ & Post-M/SE & Change M/SE & Pre-M/SE & Post-M/SE & Change M/SE & & & \\
\hline Dementia Worry & $28.39 / 0.71$ & $17.64 / 0.88$ & $-10.75 / 1.06$ & $26.45 / 0.88$ & $25.72 / 0.43$ & $-0.73 / 1.00$ & $-10.02^{* * *}$ & $-1.49^{* * *}$ & $-9.47^{* * *}$ \\
\hline Knowledge about dementia & $11.91 / 0.53$ & $13.00 / 0.64$ & $1.09 / 0.59$ & $11.37 / 0.77$ & $14.08 / 0.86$ & $2.71 / 1.08$ & -1.62 & -0.30 & -0.37 \\
\hline Cultural beliefs about dementia & $33.30 / 1.49$ & $23.72 / 0.86$ & $-9.57 / 1.73$ & $33.13 / 1.33$ & $32.31 / 0.84$ & $-0.83 / 1.29$ & $-8.75^{* * * *}$ & $-0.87^{* * * *}$ & $-8.65^{* * *}$ \\
\hline
\end{tabular}

${ }^{* * *} p<.001$. 
the increase of age, $b=1.03, p<0.01$. The treatment effect significantly became more pronounced with the increase of baseline biased belief, $\mathrm{b}=-0.39, \mathrm{p}<0.05$, which may be explained by the floor effect of baseline biased belief.

\section{Discussion}

Dementia worry is a profound phenomenon among geriatric populations internationally, including Chinese older adults. Given the negative health consequences of dementia worry, it is critical to explore evidence-based psychosocial educational interventions to ameliorate Chinese older adults' dementia worry. While promising evidence regarding psychological treatments for depression and anxiety in people with dementia or cognitive impairment exists (Orgeta, Qazi, Spector, and Orrell, 2014), it is also important to develop treatments for dementia worry in cognitively intact elders or those with minimum cognitive decline. Building on a strong body of literature supporting CBT's effectiveness for anxiety, our study contributed to the literature by specifically focusing our investigation on dementia worry using culturally adapted evidence-based treatment, like CBT, for Chinese older adults.

The results of this study provided efficacy data supporting CBT in reducing dementia worry among Chinese older adults. In comparison to no-treatment control, study outcomes indicated that modified group CBT significantly reduced older adults' dementia worry and culturally biased beliefs about dementia. More importantly, this study revealed statistically significant and large between-group effect size for dementia worry and biased beliefs about dementia, indicating clinically meaningful effects of modified group CBT for Chinese older adults' dementia worry.

Consistent with our theoretical framework, reduced biased cultural belief about dementia - a concept related to severity of consequences seemed to affect participants' dementia worry. This is supported by the identified significant changes on cultural beliefs about dementia and dementia worry among the treatment group. It is likely that reduction in participants' false belief consequently decreased their dementia worry. Future trials are encouraged to include larger sample of participants and more assessment points to test possible change mechanisms.

Modified group CBT did not improve participants' knowledge about dementia in comparison to no-treatment control, despite an upward trend in the treatment group and a downward pattern in the control group. Additionally, having more knowledge may not lessen or increase dementia anxiety, depending on how people absorb it. One's personality that varies from overwhelming pessimistic to optimistic and social support to deal with a foreseen disease tend to influence their mental state more than the mere level of dementia knowledge.

A couple of observations and reflections during our study are worth noting for future trials and treatment implementation. First, many participants $(n=16,42 \%)$ in the no-treatment control group dropped out during the study. Most participants (13 out of 16, 82\%) did not provide a reason or indicated that they were no longer interested in participating. In contrast, high retention rate was observed among participants receiving modified group CBT. One plausible reason is that the modified group CBT contains contents and activities that are engaging and popular among Chinese older adults, like Taichi. Therefore, we suggest future clinical trials and efforts involve psychosocial support for Chinese older adults to include those culturally and developmentally engaging activities for optimal outcome.

Given the high dropout rate in the control group, it is also worth noting that it might simply be due to better engagement and stronger therapeutic alliance that lead to CBT's effectiveness for dementia worry among older adults, rather than actual CBT techniques. However, based on existing psychotherapy research (Laska, Gurman, and Wampold, 2014; Wampold, 2015), it is reasonable to expect that both common factors (e.g., therapeutic alliance, engagement) and specific techniques (i.e., specific CBT techniques) that contribute to the effectiveness of CBT for Chinese older adults' dementia worry.

Second, heterogeneity test revealed that treatment effect on cultural beliefs about dementia was negatively associated with one's age. While psychoeducation can be a powerful approach in providing psychosocial support for Chinese older adults' dementia worry, age needs to be an important factor to consider when delivering psychoeducation. There is an embedded assumption of older people as wiser in Chinese culture. Therefore, young mental health service providers may find it challenging to provide psychoeducation to Chinese older adults who are deemed wiser and more experienced in their culture. In addition, we found the treatment effect of modified group CBT on biased belief decreases as a participant's age increases. It is likely because of the Chinese culture that links longevity with wisdom. As Chinese become older, they are regarded as wiser due to enriched life experiences. Consequently, older Chinese may be less receptive to their younger counterparts when being challenged on their biased beliefs about dementia.

A few limitations of this study need to be noted. Given the nature and small sample size of this current study, the limited statistical power may have led to a high risk of experiencing a type II error. Nor were we able to conduct any mediation analysis to identify specific mechanisms (e.g., Taichi component) responsible for dementia worry change. We were unable to assess treatment contamination, where participants in the control group may learn about the intervention from the treatment group. Although the intent-to-treat analysis reflects the practical scenario and acknowledges noncompliance and protocol deviation (Gupta, 2011), this approach tends to underestimate the treatment effect on outcome, especially on knowledge of dementia in our study. In addition, prevention of dropout in the control group and the inclusion of follow up assessments after intervention calls for future investigation.

As dementia is a global public health concern, seeking effective prevention and intervention efforts to increase the awareness of dementia and reduce negative beliefs about dementia grounded in cultural norms has become a critical task for service professionals and policymakers. As China has the largest population affected by dementia in the world (Prince et al., 2013), helping professionals not limited to psychologists, social workers, counselors, and psychiatrists in China face the pressing need to develop and test culturally based interventions to reduce older adults' worry over dementia. Particularly, efforts are needed to study and serve the needs of the rural older Chinese populations, who lag behind in many aspects compared to their counterparts living in Shanghai, a place with heightened awareness of dementia and rich social resources. This study represents as an effort in this direction, attempting to alleviate older adults' concerns of developing dementia by providing psychoeducation, cognitive regulation, behavioral skills training, and access to a culturally accepted exercise.

\section{Funding}

This work was supported by the National Social Science Fund of China [Grant Number 17BSH118].

\section{Author statement}

Authors state that we have all made meaningful contribution to the manuscript to be listed as a (co)author of the study.

We acknowledge the support from the National Social Science Fund of China [Grant Number 17BSH118], which partially funded our study. The funding source provided salary support for study staff, participants' incentives, and study investigator's effort. We report no conflict of interests. 


\section{Declaration of Competing Interests}

We have no conflict of interest to declare.

\section{Acknowledgment}

There is no acknowledgement for this study.

\section{Supplementary materials}

Supplementary material associated with this article can be found, in the online version, at doi:10.1016/j.jad.2020.05.054.

\section{References}

Barsky, A.J., Ahern, D.K., 2004. Cognitive behavior therapy for hypochondriasis: a ran domized controlled trial. JAMA 291 (12), 1464-1470.

Beck, A., T., Emery, Greenberg, R., 1985. Anxiety Disorders and Phobias: A Cognitive Perspective. Basic Books, New York.

...Blendon, R., Benson, J., Wikler, E., Weldon, K., Baumgart, M., Jansen, S., Georges, J., 2011. Five-country survey of public experiences, attitudes and beliefs concerning Alzheimer's disease and the value of a diagnosis. Alzheimer's Dement.: J. Alzheimer's Assoc. 7 (4), e50.

Boswell, J.F., 2013. Intervention strategies and clinical process in transdiagnostic cognitive-behavioral therapy. Psychotherapy 50 (3), 381-386.

Boswell, J.F., Iles, B.R., Gallagher, M.W., 2017. Behavioral activation strategies in cognitive-behavioral therapy for anxiety disorders. Psychotherapy 54 (2), 231-236.

Carpenter, J.K., Andrews, L.A., Witcraft, S.M., Powers, M.B., Smits, J.A., Hofmann, S.G., 2018. Cognitive behavioral therapy for anxiety and related disorders: A meta-analysis of randomized placebo-controlled trials. Depress. Anxiety 35 (6), 502-514.

Carpenter, B.D., Balsis, S., Otilingam, P.G., Hanson, P.K., Gatz, M., 2009. The Alzheimer's disease knowledge scale: development and psychometric properties. Gerontologist 49 (2), 236-247.

...Chan, K.Y., Wang, W., Wu, J.J., Liu, L., Theodoratou, E., Car, J., Rudan, I., 2013. Epidemiology of Alzheimer's disease and other forms of dementia in China, 1990-2010: a systematic review and analysis. Lancet North Am. Ed. 381 (9882), 2016-2023.

Chang, Y.P., Schneider, J.K., Sessanna, L., 2011. Decisional conflict among Chinese family caregivers regarding nursing home placement of older adults with dementia. J. Aging Stud. 25 (4), 436-444.

Chen, C.P., 1995. Counseling applications of RET in a Chinese cultural context. J. Ration. Emot. Cogn. Behav. Ther. 13 (2), 117-129.

Chen, S.W., Davenport, D.S., 2005. Cognitive-behavioral therapy with Chinese American clients: cautions and modifications. Psychother.: Theor. Res. Pract. Train. 42 (1), 101-110.

...Clark, D.M., Salkovskis, P.M., Hackmann, A., Wells, A., Fennell, M., Ludgate, J., Gelder, M., 1998. Two psychological treatments for hypochondriasis: a randomised controlled trial. Br. J. Psychiatry 173 (3), 218-225.

Cooper, C., Balamurali, T.B.S., Selwood, A., Livingston, G., 2007. A systematic review of intervention studies about anxiety in caregivers of people with dementia. Int. J. Geriatr. Psychiatry 22 (3), 181-188.

Cutler, S.J., Hodgson, L.G., 1996. Anticipatory dementia: a link between memory appraisals and concerns about developing Alzheimer's disease. Gerontologist 36 (5), 657-664.

Cuijpers, P., Sijbrandij, M., Koole, S., Huibers, M., Berking, M., Andersson, G., 2014. Psychological treatment of generalized anxiety disorder: a meta-analysis. Clin. Psychol. Rev, 34 (2), 130-140.

Fu, R., \& Holmer, H. K. (2015). Change score or followup score? A empirical evaluation of the impact of choice of mean difference estimates [internet]. Rockville (MD): Agecy for Health Research and Quality (US); Report No.: 15-EHC016-EF.

Gulpers, B., Ramakers, I., Hamel, R., Köhler, S., Voshaar, R.O., Verhey, F., 2016. Anxiety as a predictor for cognitive decline and dementia: a systematic review and metaanalysis. Am. J. Geriatr. Psychiatry 24 (10), 823-842.

Guo, Z., 2003. Response to dementia-related illness: an ethnographic study in a Chinese American community. In: Zhan, L. (Ed.), Asian Americans, Vulnerable Populations, Model Interventions, and Clarifying Agendas. Jones Bartlett Publishers, pp. 253-267.

Guo, F., Hanley, T., 2015. Adapting cognitive behavioral therapy to meet the needs of Chinese clients: opportunities and challenges. PsyCh J. 4 (2), 55-65.

Gupta, S.K., 2011. Intention-to-treat concept: a review. Perspect. Clin. Res. 2 (3), 109-112.

Hays, P.A., 2009. Integrating evidence-based practice, cognitive-behavior therapy, and multicultural therapy: ten steps for culturally competent practice. Prof. Psychol.: Res. Pract. 40 (4), 354.

Hedman, E., Ljótsson, B., Andersson, E., Rück, C., Andersson, G., Lindefors, N., 2010. Effectiveness and cost offset analysis of group CBT for hypochondriasis delivered in a psychiatric setting: an open trial. Cogn. Behav. Ther. 39 (4), 239-250.

Hofmann, S.G., Smits, J.A.J., 2008. Cognitive-behavioral therapy for adult anxiety disorders: a meta-analysis of randomized placebo-controlled trials. J. Clin. Psychiatry 69 (4), 621-632

Hui, C., Zhihui, Y., 2017. Group cognitive behavioral therapy targeting intolerance of uncertainty: a randomized trial for older Chinese adults with generalized anxiety disorder. Aging Ment. Health 21 (12), 1294-1302.

Hwang, W.C., 2006. The psychotherapy adaptation and modification framework: application to Asian Americans. Am. Psychol. 61 (7), 702.

..Hwang, W.C., Myers, H.F., Chiu, E., Mak, E., Butner, J.E., Fujimoto, K., Miranda, J., 2015. Culturally adapted cognitive-behavioral therapy for Chinese Americans with depression: a randomized controlled trial. Psychiatr. Serv. 66 (10), 1035-1042.

Kessler, E.M., Bowen, C.E., Baer, M., Froelich, L., Wahl, H.W., 2012. Dementia worry: a psychological examination of an unexplored phenomenon. Eur. J. Ageing 9 (4), 275-284.

Kinzer, A., Suhr, J.A., 2016. Dementia worry and its relationship to dementia exposure, psychological factors, and subjective memory concerns. Appl. Neuropsychol.: Adult 23 (3), 196-204.

Laditka, J.N., Laditka, S.B., Liu, R., Price, A.E., Wu, B., Friedman, D.B., Logsdon, R.G., 2011. Older adults' concerns about cognitive health: Commonalities and differences among six United States ethnic groups. Ageing Soc. 31 (7), 1202-1228.

Lam, T., Sun, K., Chan, H., Lau, C., Lam, K., Sanson-Fisher, R., 2019. Perceptions of Chinese towards dementia in Hong Kong - diagnosis, symptoms and impacts. Int. J. Environ. Res. Public Health 16 (1), 128.

Laska, K.M., Gurman, A.S., Wampold, B.E., 2014. Expanding the lens of evidence-based practice in psychotherapy: a common factors perspective. Psychotherapy 51 (4), 467.

Leong, F.T., 1986. Counseling and psychotherapy with Asian-Americans: review of the literature. J. Couns. Psychol. 33, 196-222.

...Li, C., Neugroschl, J., Umpierre, M., Martin, J., Huang, Q., Zeng, X., Sano, M., 2016. Beijing version of the montreal cognitive assessment for elderly Chinese Americans. Alzheimer's Dement.: J. Alzheimer's Assoc. 12 (7), P567.

Liang, R.J., An, Q.L., 2018. An intervention study on the aging anxiety of people about Alzheimer's disease in nursing institution: taking the "Brain Gym" program of Shanghai D and M welfare institute as an example. Soc. Work Manag. 18, 21-32 (paper in Chinese).

Molden, J., Maxfield, M., 2017. The impact of aging stereotypes on dementia worry. Eur. J. Ageing 14 (1), 29-37.

Norton, P.J., 2012. Group cognitive-behavioral therapy of anxiety: A transdiagnostic treatment manual.New. Guilford Press, York, NY.

Olatunji, B.O., Kauffman, B.Y., Meltzer, S., Davis, M.L., Smits, J.A., Powers, M.B., 2014 Cognitive-behavioral therapy for hypochondriasis/health anxiety: a meta-analysis of treatment outcome and moderators. Behav. Res. Ther. 58, 65-74.

Orgeta, V., Qazi, A., Spector, A.E., Orrell, M., 2014. Psychological treatments for depression and anxiety in dementia and mild cognitive impairment. Cochrane Database Syst. Rev.(1).

Petkus, A.J., Reynolds, C.A., Wetherell, J.L., Kremen, W.S., Pedersen, N.L., Gatz, M., 2016. Anxiety is associated with increased risk of dementia in older Swedish twins. Alzheimer's Dement. 12 (4), 399-406.

Prince, M., Bryce, R., Albanese, E., Wimo, A., Ribeiro, W., Ferri, C.P., 2013. The global prevalence of dementia: a systematic review and metaanalysis. Alzheimer's Dement. 9 (1), 63-75.

Puma, M. J., Olsen, R. B., Bell, S. H., \& Price, C. (2009). What to do when data are missing in group randomized controlled trials. NCEE 2009-0049. National Center for Education Evaluation and Regional Assistance. Retrieved from:https://files.eric.ed. gov/fulltext/ED511781.pdf.

Rathod, S., Phiri, P., Naeem, F., 2019. An evidence-based framework to culturally adapt cognitive behavior therapy. Cognit. Behav. Ther. 12 (e10), 1-15.

Roberts, J.S., Connell, C.M., 2000. Illness representations among first-degree relatives of people with Alzheimer disease. Alzheimer Dis. Assoc. Disord. 14 (3), 129-136.

Royston, P., White, I.R., 2011. Multiple imputation by chained equations (MICE): implementation in Stata. J. Stat. Softw. 45 (4), 1-20.

Seignourel, P.J., Kunik, M.E., Snow, L., Wilson, N., Stanley, M., 2008. Anxiety in dementia: a critical review. Clin. Psychol. Rev. 28 (7), 1071-1082.

Shi, Y., Sun, F., Liu, Y., Marsiglia, F.F., 2018. Perceived threat of Alzheimer's disease and related dementias among older Chinese Americans in subsidized housing: through a cultural lens. Dementia 1471301218805901.

...Spector, A., Charlesworth, G., King, M., Lattimer, M., Sadek, S., Marston, L., Orrell, M., 2015. Cognitive-behavioural therapy for anxiety in dementia: pilot randomised controlled trial. Br. J. Psychiatry 206 (6), 509-516.

Sun, F., Gao, X., Coon, D.W., 2013. Perceived threat of Alzheimer's disease among Chinese American older adults: the role of Alzheimer's disease literacy. J. Gerontol. Ser. B: Psychol. Sci. Soc. Sci. 70 (2), 245-255.

...Tadros, G., Ormerod, S., Dobson-Smyth, P., Gallon, M., Doherty, D., Carryer, A., Kingston, P., 2013. The management of behavioural and psychological symptoms of dementia in residential homes: does Tai Chi have any role for people with dementia. Dementia 12 (2), 268-279.

Tung, M., 1991. Insight-oriented psychotherapy and the Chinese patients. Am. J. Orthopsychiatry 61 (2), 186-194.

Wampold, B.E., 2015. How important are the common factors in psychotherapy? An update. World Psychiatry 14 (3), 270-277.

Wang, C., Bannuru, R., Ramel, J., Kupelnick, B., Scott, T., Schmid, C.H., 2010. Tai Chi on psychological well-being: systematic review and meta-analysis. BMC Complement. Altern. Med. 10 (1), 23.

Wang, C., Collet, J.P., Lau, J., 2004. The effect of Tai Chi on health outcomes in patients with chronic conditions: a systematic review. Arch. Intern. Med. 164 (5), 493-501.

Warwick, H., 2004. Treatment of health anxiety. Psychiatry 3 (6), 80-83.

Warwick, H.M.C., Salkovskis, P.M., 1990. Hypochondriasis. Behav. Res. Ther. 28 (2), 105-117.

Watts, S.E., Turnell, A., Kladnitski, N., Newby, J.M., Andrews, G., 2015. Treatment-asusual (TAU) is anything but usual: a meta-analysis of CBT versus TAU for anxiety and depression. J. Affect. Disord. 175, 152-167.

Wayne, P.M., Walsh, J.N., Taylor-Piliae, R.E., Wells, R.E., Papp, K.V., Donovan, N.J., Yeh, 
G.Y., 2014. Effect of Tai Chi on cognitive performance in older adults: systematic review and meta-analysis. J. Am. Geriatr. Soc. 62 (1), 25-39.

Wilson, R.S., Begeny, C.T., Boyle, P.A., Schneider, J.A., Bennett, D.A., 2011. Vulnerability to stress, anxiety, and development of dementia in old age. Am. J. Geriatr. Psychiatry 19 (4), 327-334.

Yu, J., Li, J., Huang, X., 2012. The Beijing version of the montreal cognitive assessment as a brief screening tool for mild cognitive impairment: a community-based study. BMC Psychiatry 12, 156.

Yu, H., Wu, L., Chen, S., Wu, Q., Yang, Y., Edwards, H., 2016. Caregiving burden and gain among adult-child caregivers caring for parents with dementia in China: the partial mediating role of reciprocal filial piety. Int. Psychogeriatr. 28 (11), 1845-1855.

...Zeng, F., Xie, W.T., Wang, Y.J., Luo, H.B., Shi, X.Q., Zou, H.Q., Lian, Y., 2015. General public perceptions and attitudes toward Alzheimer's disease from five cities in China. J. Alzheimer's Dis. 43 (2), 511-518.

Zhang, Y., 2018. Governing dementia: a historical investigation of the power of states and professionals in the conceptualization of dementia in China. Cult. Med. Psychiatry 42 (4), 862-892. 